\title{
RESTRICTIONS OF ANALYTIC FUNCTIONS. II
}

\section{MARVIN ROSENBLUM AND JAMES ROVNYAK ${ }^{1}$}

\begin{abstract}
An isometric expansion is derived which recaptures any
$H^{2}$ function from a restriction of its boundary function to a Borel set.
\end{abstract}

1. Introduction. Let $\Delta$ be a Borel subset of the real line such that neither $\Delta$ nor its complement $\Delta^{c}$ is a Lebesgue null set. Let $f(x)$ be a complex valued measurable function on $\Delta$. In [4] we derived conditions for the existence of a function $F(z)$ in $H^{2}$ whose boundary function $F(x)$ agrees with $f(x)$ a.e. on $\Delta$. General methods for recapturing $F(z)$ from a knowledge of $f(x)$ have been given by Golusin and Krylov [1] and Patil [3]. When $\Delta$ is an interval, say $\Delta=(0, \infty)$, there is a more refined theory due to van Winter [9] which shows how $F(z)$ can be recaptured from $f(x)$ by means of reciprocal formulas of the Mellin form. Closely related results were obtained independently by Krein and Nudel'man [2]. See also Steiner [7].

We give a new derivation of the Mellin representation of $H^{2}$. Our main purpose, however, is to extend the representation to the case where $\Delta$ is a general Borel set. The proof uses Cayley inner functions and the methods of $[5]$ to reduce the general result to the special case where $\Delta$ is an interval.

2. Mellin representation of $H^{2}$ functions. By $H^{2}$ we mean the space of functions $F(z)$ analytic for $y>0$ such that

$$
\sup _{y>0} \int_{-\infty}^{+\infty}|F(x+i y)|^{2} d x<\infty \text {. }
$$

Theorem 1 (van Winter [9]). (i) If $\mathcal{F}(t)$ is a measurable function on $(-\infty, \infty)$ such that

Received by the editors June 12, 1974.

AMS (MOS) subject classifications (1970). Primàry 30A78, 30A88; Secondary 42A76, 47B99.

Key words and phrases. $H^{2}$, Cayley inner function, boundary function, Mellin transform.

1 This research was supported by NSF Grant GP-31483X. The first author also received support from the Center for Advanced Studies of the University of Virginia. 


$$
\int_{-\infty}^{+\infty}\left(1+e^{2 \pi t}\right)|\mathcal{F}(t)|^{2} d t<\infty
$$

then

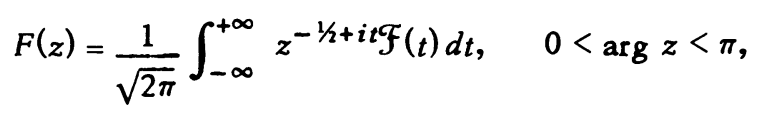

defines a function in $y^{2}$ whose boundary function $F(x)=F(x+i 0)$ satisfies

$$
\begin{aligned}
& \int_{0}^{\infty}|F(x)|^{2} d x=\int_{-\infty}^{+\infty}|\mathcal{F}(t)|^{2} d t, \\
& \int_{-\infty}^{0}|F(x)|^{2} d x=\int_{-\infty}^{+\infty} e^{2 \pi t}|\mathcal{F}(t)|^{2} d t,
\end{aligned}
$$

and indeed, more generally,

$$
\int_{0}^{+\infty}\left|F\left(r e^{i \theta}\right)\right|^{2} d r=\int_{-\infty}^{+\infty} e^{2 \theta t}|\mathcal{F}(t)|^{2} d t
$$

for each fixed $\theta, 0 \leq \theta \leq \pi$. Conversely, if $F(z)$ is an $H^{2}$ function, there exists an essentially unique function $\mathcal{F}(t)$ satisfying (1) such that (2)-(5) hold. The inversion formula

$$
\mathcal{F}(t)=\lim _{T \rightarrow \infty} \frac{1}{\sqrt{2 \pi}} \int_{1 / T}^{T}\left(r e^{i \theta}\right)^{-1 / 2+i t} F\left(r e^{i \theta}\right) e^{i \theta} d r
$$

holds for each fixed $\theta, 0 \leq \theta \leq \pi$, where convergence is in the metric of $L^{2}(-\infty, \infty)$.

(ii) Suppose $F(x) \in L^{2}(0, \infty)$, and define

$$
\mathcal{F}(t)=\lim _{T \rightarrow \infty} \frac{1}{\sqrt{2 \pi}} \int_{1 / T}^{T} x^{-1 / 2+i t} F(x) d x
$$

with convergence in the metric of $L^{2}(-\infty, \infty)$. Then there exists a function $F(z)$ in $H^{2}$ such that $F(x+i 0)=F(x)$ a.e. on $(0, \infty)$ if and only if $\mathcal{F}(t)$ satisfies (1), and in this case $F(z)$ satisfies (2)-(6).

Proof. The space $H^{2}$, regarded as a Hilbert space in the usual norm, has reproducing kernel $K_{0}(w, z)=(2 \pi i)^{-1}\left(w^{*}-z\right)^{-1}$. Let $K$ be the Hilbert space of functions

$$
G(z)=\frac{1}{\sqrt{2 \pi}} \int_{-\infty}^{+\infty} e^{t z} \mathcal{F}(t) d t, \quad 0<\operatorname{Re} z<\pi,
$$

where $\mathscr{F}(t)$ satisfies (1) and $\|G\|^{2}$ is equal to the expression in (1). Routine arguments show that $\mathcal{K}$ has reproducing kernel

$$
K(w, z)=\frac{1}{2 \pi} \int_{-\infty}^{+\infty} e^{t z} e^{t w^{*}}\left(1+e^{2 \pi t}\right)^{-1} d t
$$


Since by $[8$, p. 192]

$$
\int_{0}^{\infty} x^{s-1}(1+x)^{-1} d x=\pi \csc (\pi s), \quad 0<\operatorname{Re} s<1,
$$

we obtain

$$
K(w, z)=e^{i z / 2} K_{0}\left(e^{i w}, e^{i z}\right) e^{-i w^{*} / 2} .
$$

It follows that $F(z) \rightarrow e^{i z / 2} F\left(e^{i z}\right)$ is an isometry mapping $H^{2}$ onto $\mathcal{K}$ with inverse $G(z) \rightarrow z^{-1 / 2} G(-i \log z), 0<\arg z<\pi$. Therefore $H^{2}$ is exactly the set of functions in the upper half-plane of the form

$$
\begin{aligned}
F(z) & =z^{-1 / 2} G(-i \log z)=\frac{1}{\sqrt{2 \pi}} z^{-1 / 2} \int_{-\infty}^{+\infty} e^{-i t \log z \mathcal{F}(t) d t} \\
& =\frac{1}{\sqrt{2 \pi}} \int_{-\infty}^{+\infty} z^{-1 / 2-i t} \mathcal{F}(t) d t .
\end{aligned}
$$

Let $\mathcal{F}(t)$ and $F(z)$ be related as above, and define $G(z)$ by (8), so $G(z)$ $=e^{i z / 2} F\left(e^{i z}\right)$. Define

$$
\begin{aligned}
& G(0+i y)=\frac{1}{\sqrt{2 \pi}} \int_{-\infty}^{+\infty} e^{t(0+i y)} \mathcal{F}(t) d t, \\
& G(\pi+i y)=\frac{1}{\sqrt{2 \pi}} \int_{-\infty}^{+\infty} e^{t(\pi+i y)} \mathcal{F}(t) d t
\end{aligned}
$$

where the integrals are taken in the mean square sense. By Parseval's formula, $\lim G(x+i y)=G(0+i y)$ as $x \backslash 0$ and $\lim G(x+i y)=G(\pi+i y)$ as $x$ $>\pi$ in the metric of $L^{2}(-\infty, \infty)$. The limits hold a.e. also because $F(x)=$ $\lim F(x+i y)$ as $y>0$ nontangentially a.e. Therefore the relation

$$
G(\theta+i y)=e^{i(\theta+i y) / 2} F\left(e^{i(\theta+i y)}\right)
$$

holds not only for $0<\theta<\pi$, but also a.e. when $\theta=0, \pi$. Now if $0 \leq \theta \leq \pi$, then

$$
\int_{-\infty}^{+\infty}|G(\theta+i y)|^{2} d y=\int_{-\infty}^{+\infty} e^{-y}\left|F\left(e^{-y} e^{i \theta}\right)\right|^{2} d y=\int_{0}^{\infty}\left|F\left(r e^{i \theta}\right)\right|^{2} d r
$$

But by Parseval's formula

$$
\int_{-\infty}^{+\infty}|G(\theta+i y)|^{2} d y=\int_{-\infty}^{+\infty} e^{2 \theta t}|\mathcal{F}(t)|^{2} d t .
$$

so (3)-(5) follow. Using (8) when $0<\theta<\pi$ and (9) when $\theta=0$, $\pi$, we obtain a.e. 


$$
\begin{aligned}
\mathcal{F}(t) & =\frac{1}{\sqrt{2 \pi}} \int_{-\infty}^{+\infty} e^{-i t y} e^{-\theta t} G(\theta+i y) d y \\
& =\frac{1}{\sqrt{2 \pi}} \int_{-\infty}^{+\infty} e^{-i t y} e^{i \theta(1 / 2+i t)} e^{-1 / 2 y} F\left(e^{-y} e^{i \theta}\right) d y \\
& =\frac{1}{\sqrt{2 \pi}} \int_{0}^{\infty}\left(r e^{i \theta}\right)^{-1 / 2+i t} F\left(r e^{i \theta}\right) e^{i \theta} d r
\end{aligned}
$$

where the integrals are taken in the mean square sense. This yields (6) and completes the proof of (i). The assertions in (ii) follow directly from (i).

3. Generalization. Let $\Delta$ be a Borel subset of the real line such that neither $\Delta$ nor $\Delta^{c}$ is a Lebesgue null set. In the terminology of [5], a Cayley inner function mapping $\Delta$ on $(0, \infty)$ is any function $\xi(z)$ which is analytic and satisfies $\xi\left(z^{*}\right)=\xi(z)^{*}$ for $z \neq z^{*}$ and

(i) $\operatorname{Im} \xi(z)>0$ for $y>0$,

(ii) $\xi(x+i 0)=\xi(x-i 0)$ a.e. on $(-\infty, \infty)$, and

(iii) $\xi(x) \stackrel{\text { def }}{=} \xi(x+i 0)=\xi(x-i 0)$ satisfies $\xi(x)>0$ a.e. on $\Delta$ and $\xi(x)$ $<0$ a.e. on $\Delta^{c}$.

The general form of such a function [5, Theorem 2.2] is given by

$$
\xi(z)=-1 / \exp \left(k+\int_{\Delta} \frac{1+t z}{t-z} \frac{d t}{1+t^{2}}\right)
$$

where $k$ is a real number. We understand that some such function is chosen and held fixed in the discussion. When $\Delta=\bigcup_{1}^{r}\left(a_{j}, b_{j}\right)$ where $-\infty<a_{1}<b_{1}$ $<a_{2}<b_{2}<\cdots<a_{r}<b_{r}<\infty$, a convenient choice is

$$
\xi(z)=-1 / \exp \left(\int_{\Delta} \frac{d t}{t-z}\right)=-\prod_{1}^{r} \frac{a_{j}-z}{b_{j}-z} .
$$

If $\Delta=(0, \infty)$, then necessarily $\xi(z)=\tau z$ where $0<\tau<\infty$, and we may choose $\xi(z)=z$.

We introduce the notation

$$
l(\alpha, \beta)=\frac{\xi(\alpha)-\xi(\beta)^{*}}{\alpha-\beta^{*}} \text { and } l(t, \beta)=\frac{\xi(t)-\xi(\beta)^{*}}{t-\beta^{*}}
$$

for $a \neq \alpha^{*}, \beta \neq \beta^{*}$, and $t$ real. As noted in [5], "composition with $\xi(t)$ " is a meaningful operation in the class of a.e. defined functions on the real line. By [5, Theorem 3.3], if $f(t) \in L^{1}(-\infty, \infty)$ and $g(t) \in L^{1}(0, \infty)$, and $\alpha \neq \alpha^{*}$, $\beta \neq \beta^{*}$, then

$$
\int_{-\infty}^{+\infty} l(t, \alpha) l(t, \beta)^{*} f(\xi(t)) d t=l(\beta, \alpha) \int_{-\infty}^{+\infty} f(t) d t,
$$


and

$$
\int_{\Delta} l(t, \alpha) l(t, \beta)^{*} g(\xi(t)) d t=l(\beta, \alpha) \int_{0}^{\infty} g(t) d t
$$

where the integrals on the left are absolutely convergent.

Theorem 2. (i) If $\mathcal{F}(t)$ is a measurable function on $(-\infty, \infty)$ such that

$$
\int_{-\infty}^{+\infty}\left(1+e^{2 \pi \xi(t)}\right)|\mathcal{F}(t)|^{2} d t<\infty
$$

then

$$
F(z)=\frac{1}{\sqrt{2 \pi}} \int_{-\infty}^{+\infty} \frac{\xi(t)-\xi(z)}{t-z} \xi(z)^{-1 / 2-i \xi(t) \mathcal{G}}(t) d t
$$

defines a function in $H^{2}$ whose boundary function $F(x)=F(x+i 0)$ satisfies

$$
\int_{\Delta}|F(x)|^{2} d x=\int_{-\infty}^{+\infty}|\mathcal{F}(t)|^{2} d t
$$

and

$$
\int_{\Delta^{c}}|F(x)|^{2} d x=\int_{-\infty}^{+\infty} e^{2 \pi \xi(t)}|\mathcal{F}(t)|^{2} d t
$$

Conversely, if $F(z)$ is an $H^{2}$ function, there exists an essentially unique function $\mathcal{F}(t)$ satisfying (12) such that (13)-(15) hold. The inversion formula

$$
\text { (16) } \mathcal{F}(t)=\frac{1}{\sqrt{2 \pi}}\left(\lim _{\epsilon \backslash 0} \int_{\Delta_{+}}+\lim _{\epsilon / 0} \int_{\Delta_{-}}\right) \frac{\xi(x)-\xi(t+i \epsilon)}{x-t-i \epsilon} \xi(x)^{-1 / 2+i \xi(t+i \epsilon)} F(x) d x
$$

holds a.e. and in the metric of $L^{2}(-\infty, \infty)$, where $\Delta_{+}=\{x: \xi(x)>1\}$ and $\Delta_{-}$ $=\{x: 0<\xi(x)<1\}$.

(ii) For every $F(x) \in L^{2}(\Delta),(16)$ defines a function $\mathcal{F}(t) \in L^{2}(-\infty, \infty)$. There exists a function $F(z)$ in $H^{2}$ such that $F(x+i 0)=F(x)$ a.e. on $\Delta$ if and only if $\mathscr{F}(t)$ satisfies (12), and then (13)-(15) hold.

Proof. Let $W(t)=1+e^{2 \pi \xi(t)}$ and let $L^{2}(W)$ denote the Lebesgue space associated with the measure $W d x$ on $(-\infty, \infty)$. We first exhibit a special dense subspace of $L^{2}(W)$. Define $L^{2}\left(W_{0}\right)$ similarly for $W_{0}(t)=1+e^{2 \pi t}$. We assert that functions of the form

$$
\mathcal{F}(t)=l(t, w) \mathcal{F}_{0}(\xi(t)),
$$

where $w \neq w^{*}$ and $\mathcal{F}_{0} \in L^{2}\left(W_{0}\right)$, belong to $L^{2}(W)$ and span a dense subspace of $L^{2}(W)$. By (10) such functions belong to $L^{2}(W)$. To see that there are 
enough to span a dense subspace, choose $\mathfrak{F}_{0}(t)=\chi_{(-A, A)}(t) /\left(t-\xi(w)^{*}\right)$, where $w \neq w^{*}$ and $A>0$. It follows that $\chi\{x:|\xi(x)|<A\}(t) /\left(t-w^{*}\right)$ belongs to the set, and so density follows by routine arguments.

Next we exhibit a special dense subspace of $H^{2}$. Namely, we assert that functions of the form

$$
F(z)=l(z, w) F_{0}(\xi(z)), \quad y>0,
$$

where $w \neq w^{*}$ and $F_{0} \in H^{2}$, belong to $H^{2}$ and span a dense subspace of $H^{2}$. Assume $F_{0} \in H^{2}, w \neq w^{*}$, and define $F$ by. (18). By Cauchy's formula,

$$
F_{0}(z)=\frac{1}{2 \pi i} \int_{-\infty}^{+\infty} \frac{F_{0}(t)}{t-z} d t, \quad y>0 .
$$

By (10) the function $F(x)=l(x, w) F_{0}(\xi(x))$ is in $L^{2}(-\infty, \infty)$. We note that by a theorem of Ryff [6], $F(x)=F(x+i 0)$ is the boundary function of $F(z)$. This justifies the notation, but logically it is not needed here. It will be used later. We obtain

$$
F(z)=\int_{-\infty}^{+\infty} \frac{F(z)}{t-z} d t, \quad y>0,
$$

from the Cauchy representation of $F_{0}(z)$ using (10). Thus $F \in H^{2}$. Choosing $F_{0}(z)=1 /\left[z-\xi(w)^{*}\right]$, we obtain $F(z)=1 /\left(z-w^{*}\right)$, and so density follows.

Next we show that (13) defines an isometry $U_{+}: \mathfrak{F}(t) \rightarrow F(z)$ mapping $L^{2}(W)$ onto $H^{2}$. Straightforward estimates show that for each fixed $z$ in the upper half-plane the integral in (13) is a continuous linear functional of $\mathcal{F}$ on $L^{2}(W)$. By what was proved above, it therefore suffices to check that

$$
\begin{aligned}
\left\langle l(t, \alpha) \mathcal{F}_{0}(\xi(t)),\right. & \left.l(t, \beta) \Theta_{0}(\xi(t))\right\rangle_{L^{2}(w)} \\
& =\left\langle l(z, \alpha) F_{0}(\xi(z)), l(z, \beta) G_{0}(\xi(z))\right\rangle_{H^{2}}
\end{aligned}
$$

and

$$
\begin{aligned}
& l(z, w) F_{0}(\xi(z)) \\
& =\frac{1}{\sqrt{2 \pi}} \int_{-\infty}^{+\infty} l(t, z)^{*} \xi(z)^{-1 / 2-i \xi(t)} l(t, w) \mathcal{F}_{0}(\xi(t)) d t, \quad y>0,
\end{aligned}
$$

for any nonreal numbers $\alpha, \beta, w$ and any $\mathcal{F}_{0}, \varrho_{0} \in L^{2}\left(W_{0}\right)$ and $F_{0}, G_{0} \in H^{2}$ which are connected by

$$
F_{0}(z)=\frac{1}{\sqrt{2 \pi}} \int_{-\infty}^{+\infty} z^{-1 / 2-i t \mathcal{F}_{0}}(t) d t, \quad G_{0}(z)=\frac{1}{\sqrt{2 \pi}} \int_{-\infty}^{+\infty} z^{-1 / 2-i t \varrho_{0}}(t) d t
$$

for $y>0$. Here, of course, implicit use is made of Theorem 1 in knowing 
that $F_{0}$ and $G_{0}$ exist given $\mathcal{F}_{0}$ and $\mathcal{G}_{0}$, and conversely any $F_{0}$ and $G_{0}$ arise from some $\mathcal{F}_{0}$ and $\zeta_{0}$. In fact, Theorem 1 combined with (10) yields (19). We obtain (20) from (10) and (21). The details are left to the reader.

Let $\mathcal{C}$ be the Hilbert space with reproducing kernel $l(z, w), w \neq w^{*}, z$ $\neq z^{*}$. The elements of $\mathcal{C}$ are functions separately analytic for $y>0$ and $y<0$. We see from (10) and (11) that the linear transformations

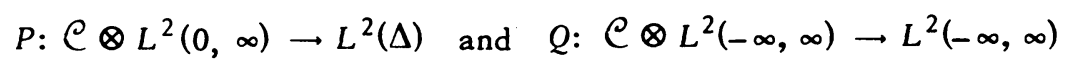

specified by

$$
P: l(z, w) \otimes f(t) \rightarrow l(t, w) f(\xi(t)) \text { and } Q: l(z, w) \otimes g(t) \rightarrow l(t, w) g(\xi(t)),
$$

where $w$ is any nonreal number, $f \in L^{2}(0, \infty), g \in L^{2}(-\infty, \infty)$, and $\otimes$ denotes the Hilbert space tensor product, are isometric isomorphisms. Next we place $\mathcal{Q} \otimes L^{2}(0, \infty)$ and $\mathcal{C} \otimes L^{2}(-\infty, \infty)$ in linear isometric correspondence by $I \otimes M$, where $M$ is the Mellin transform, so

$$
I \otimes M: l(z, w) \otimes f(t) \rightarrow l(z, w) \otimes \underset{T \rightarrow \infty}{\lim _{T \rightarrow \infty}} \frac{1}{\sqrt{2 \pi}} \int_{1 / T}^{T} x^{-1 / 2+i t} f(x) d x
$$

and

$$
(I \otimes M)^{-1}: l(z, w) \otimes g(t) \rightarrow l(z, w) \otimes \underset{T \rightarrow \infty}{1 . \mathrm{i} \cdot \mathrm{m}} \cdot \frac{1}{\sqrt{2 \pi}} \int_{-T}^{+T} t^{-1 / 2-i x} g(x) d x
$$

for each $f \in L^{2}(0, \infty)$ and $g \in L^{2}(-\infty, \infty)$.

By construction $U=P(I \otimes M)^{-1} Q^{-1}$ maps $L^{2}(-\infty, \infty)$ isometrically onto $L^{2}(\Delta)$. Note that $L^{2}(W)$ is contained in $L^{2}(-\infty, \infty)$ and the inclusion mapping is bounded by 1 . We assert that if $\mathfrak{F} \in L^{2}(W), U_{+}: \mathcal{F}(t) \rightarrow F(z)$, and $U: \mathfrak{F}(t) \rightarrow F_{\Delta}(x)$, then $F_{\Delta}(x)$ is the restriction to $\Delta$ of the boundary function $F(x)=F(x+i 0)$ of $F(z)$. It is enough to check this for a set which is dense in $L^{2}(W)$. It is true for functions of the.form (17) by direct calculation. The general case follows by linearity and continuity. We now have the diagram

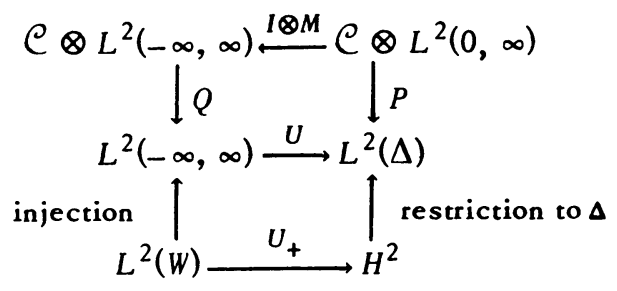

where $I \otimes M, P, Q, U$, and $U_{+}$are isometric isomorphisms.

Now if $\mathcal{F} \in L^{2}(W)$ and if $F$ in $H^{2}$ is the corresponding function given by (13), then because $U_{+}$and $U$ are unitary 


$$
\int_{-\infty}^{+\infty}|F(t)|^{2} d t=\int_{-\infty}^{+\infty}\left[1+e^{2 \pi \xi(t)}\right]|\mathcal{F}(t)|^{2} d t
$$

and

$$
\int_{\Delta}|F(t)|^{2} d t=\int_{-\infty}^{+\infty}|\mathcal{F}(t)|^{2} d t
$$

so (14) and (15) hold.

The inversion formula (16) involves an explicit calculation of $U^{-1}$, which we give next. If $\mathcal{F} \in L^{2}(-\infty, \infty)$, then

$$
\mathcal{F}(t)=\lim _{\epsilon>0} \frac{\epsilon}{\pi} \int_{-\infty}^{+\infty} \frac{\mathcal{F}(x)}{(x-t)^{2}+\epsilon^{2}} d x
$$

a.e. and in the metric of $L^{2}(-\infty, \infty)$. We assert that if $U \mathcal{F}=F$, then

$$
\begin{aligned}
& \frac{\epsilon}{\pi} \int_{-\infty}^{+\infty} \frac{\mathcal{F}(x)}{(x-t)^{2}+\epsilon^{2}} d x=\frac{1}{\sqrt{2 \pi}} \int_{\Delta_{+}} l(x, t+i \epsilon)^{*} \xi(x)^{-1 / 2+i \xi(t+i \epsilon)} F(x) d x \\
& +\frac{1}{\sqrt{2 \pi}} \int_{\Delta_{-}} l(x, t-i \epsilon)^{*} \xi(x)^{-1 / 2+i \xi(t-i \epsilon)} F(x) d x
\end{aligned}
$$

for $t$ real, $\epsilon>0$. Now for fixed $t$ and $\epsilon$, the left side of (22) equals

$$
\begin{aligned}
\frac{1}{2 \pi i} \int_{-\infty}^{+\infty} \mathcal{F}(x)[(x- & \left.t-i \epsilon)^{-1}-(x-t+i \epsilon)^{-1}\right] d x \\
= & \frac{1}{2 \pi i}\left\langle U^{-1} F,(x-t+i \epsilon)^{-1}-(x-t-i \epsilon)^{-1}\right\rangle_{L^{2}}(-\infty, \infty) \\
& =\frac{1}{2 \pi i}\left\langle F, U(x-t+i \epsilon)^{-1}-U(x-t-i \epsilon)^{-1}\right\rangle_{L^{2}(\Delta)}
\end{aligned}
$$

where here and in the rest of the proof $x$ is used as a dummy variable. We can derive (22) and consequently (16) by calculating $U(x-t+i \epsilon)^{-1}$ and $U(x-t-i \epsilon)^{-1}$. We find that

$$
Q^{-1}:(x-t+i \epsilon)^{-1} \rightarrow l(z, t+i \epsilon) \otimes\left[x-\xi(t+i \epsilon)^{*}\right]^{-1},
$$

$(I \otimes M)^{-1} Q^{-1}:(x-t+i \epsilon)^{-1} \rightarrow-i \sqrt{2 \pi} l(z, t+i \epsilon) \otimes x^{-1 / 2-i \xi(t+i \epsilon)^{*}} \chi_{(1, \infty)}(x)$, and

$$
U:(x-t+i \epsilon)^{-1} \rightarrow\left\{\begin{array}{l}
-i \sqrt{2 \pi} l(x, t+i \epsilon) \xi(x)^{-1 / 2-i \xi(t+i \epsilon)^{*}} \text { on } \Delta_{+}, \\
0 \text { on } \Delta_{-} .
\end{array}\right.
$$

Similarly

$$
U:(x-t-i \epsilon)^{-1} \rightarrow\left\{\begin{array}{l}
0 \quad \text { on } \Delta_{+}, \\
i \sqrt{2 \pi} l(x, t-i \epsilon) \xi(x)^{-1 / 2-i \xi(t-i \epsilon)^{*}} \text { on } \Delta_{-} .
\end{array}\right.
$$


This implies (22) and completes the proof of (i). The assertions in (ii) are evident from our constructions.

\section{REFERENCES}

1. G. M. Golusin and W. J. Krylov, Generalization of a formula of Carleman and its application to analytic continuation, Mat. Sb. 40 (1933), 144-149.

2. M. G. Krein and P. Ja. Nudel'man, Certain new problems for functions of a Hardy class and for continual families of functions with double orthogonality, Dokl. Akad. Nauk SSSR 209 (1973), 537-540= Soviet Math. Dokl. 14 (1973), 435-439. MR 47 \#9156.

3. D. J. Patil, Representation of $H^{p}$-functions, Bull. Amer. Math. Soc. 78 (1972), 617-620. MR 45 \#7069.

4. M. Rosenblum and J. Rovnyak, Restrictions of analytic functions. I, Proc. Amer. Math. Soc. 48 (1975), 113-119.

5. - Cayley inner functions and best approximation, J. Approximation Theory (to appear).

6. J. V. Ryff, Subordinate $H^{p}$ functions, Duke Math. J. 33 (1966), 347-354. MR 33 \#289.

7. A. Steiner, Zum Mechanismus der Quasianalytizität gewisser Randfunktionen auf endlichen Intervallen, Ann. Acad. Sci Fenn. Ser. A I No. 459 (1970). MR 41 \#8681.

8. E. C. Titchmarsh, Theory of Fourier integrals, 2nd ed., Oxford Univ. Press, New York, 1948.

9. C. van Winter, Fredholm equations on a Hilbert space of analytic functions, Trans. Amer. Math. Soc. 162 (1971), 103-139.

DEPARTMENT OF MATHEMATICS, UNIVERSITY OF VIRGINIA, CHARLOTTESVILLE, VIRGINIA 22903 